\title{
LAS COMPETENCIAS EMOCIONALES EN LOS PROCESOS DE INSERCIÓN LABORAL
}

\author{
THE EMOTIONAL COMPETENCES IN THE JOB FINDING PROCES
}

\author{
Núria Pérez Escoda* \\ Universidad de Barcelona \\ Anna Ribera Cos** \\ Ayuntamiento de Igualada
}

\begin{abstract}
RESUMEN
Este trabajo presenta un estudio basado en la evaluación y desarrollo de las competencias emocionales en los procesos de orientación y formación de personas desempleadas. Se presenta una experiencia realizada en el marco de una investigación entre el Centro IG-NOVA Ocupació de l'Ajuntament d'Igualada y el GROP (Grup de Recerca en Orientació Psicopedagògica) de la Universidad de Barcelona. Esta investigación muestra como el conocimiento y desarrollo de las competencias emocionales permite afrontar con mayor eficacia los procesos de inserción laboral. Se ha aplicado el (QDE-R) cuestionario para la evaluación de las competencias emocionales de adultos a un grupo de 46 personas adultas en situación de desempleo y con un nivel de formación medio-bajo. A partir de los resultados se exponen las aplicaciones derivadas de esta evaluación para un grupo de desempleados usuarios del Servicio Local de Ocupación.
\end{abstract}

Palabras clave: orientación profesional, competencias emocionales, educación emocional, evaluación de necesidades, formación profesional, búsqueda de empleo.

\begin{abstract}
This paper presents a study based on the evaluation and later development of the emotional competences in career guidance processes for jobseekers. This study has been carried out within research framework done by the Center IG-NOVA Ocupació of the council of Igualada and the GROP (Grup de Recerca in Orientació Psicopedagògica) (Group of Research in Psychopedagogic Guidance) of the University of Barcelona. This study shows how knowledge and development of emotional competen-
\end{abstract}

*. Profesora Titular del Departamento MIDE (Métodos de investigación y Diagnóstico en educación) de la Facultad de Pedagogia de la Universidad de Barcelona. Sus líneas de investigación son la educación emocional y la orientación profesional. Correo-e: nperezescoda@ub.edu.

**. Técnica de ocupación de IG-NOVA Ocupació del Ayuntamieto de Igualada. Sus líneas de investigación se centran en el desarrollo de competencias emocionales en la orientación y la formación para el empleo. 
ces allow to more effectively face the processes involved in job seeking. The (QDE-R) inventory was used to evaluate emotional competences in a group of 46 unemployed adults with a lower to middle level of education. All 46 jobseekers were registered within the local unemployment agency. The QDE-R measures five dimensions of the emotional competences: emotional conscience, emotional regulation, emotional autonomy, social skills, lifelong-learning skills and well-being. The results provided with an outline to support the abovementioned jobseekers group. Such jobseekers support was provided for both individuals and the whole group. Whenever individual support was provided, the focus was on those skills more likely to be developed by the individual. At the same time, there was also a strong emphasis to enhance those previously existing skills in order to help individuals in their job-seeking process. The average score of the group can provide with what dimensions must be prioritized when designing a group development program.

Key words: career guidance, emotional competences inventory, emotional competences, emotional education, emotional literacy, vocational training, job search.

\section{Introducción}

Actualmente, nuestro país acusa una importante y continuada pérdida de empleos en el marco de una crisis económica mundial. En este contexto las acciones de formación profesional ocupacional y orientación para la inserción laboral cobran una especial importancia.

Tradicionalmente en las acciones de formación para el empleo se utilizan herramientas y recursos encaminados a la obtención de competencias técnico-profesionales imprescindibles en una profesión u ocupación. Entre ellas se encuentra el dominio de conocimientos básicos y especializados, dominio de las habilidades y de técnicas necesarias en una profesión, capacidad de organización, coordinación, gestión del entorno, trabajo en equipo, adaptación e innovación (Alberici y Serreri, 2005; Bisquerra y Pérez, 2007; Le Boterf, 2001). Sin embargo recientemente y como demanda creciente en el entorno laboral, se ha constatado la necesidad de introducir en la formación y orientación profesional actividades encaminadas al desarrollo de competencias genéricas o transversales (Cherniss, 2000, Fernández Vázquez, 2005, Hué, 2003, Valls, 2007). También denominadas participativas, personales, relacionales, interpersonales, socio-emocionales, etc.

Por otro lado, numerosas investigaciones destacan el impacto que el desempleo produce en las personas afectadas. No sólo afecta a las personas económicamente, sino también psicológica y socialmente en diversos grados (Pérez, 1996). Destacan repercusiones como: baja autoestima, disminución de las relaciones sociales, sentimiento de impotencia, frustración, autoculpa, inseguridad, sentimiento de desamparo, ira y resentimiento, aislamiento, empeoramiento de la salud, cambios en los hábitos de comportamiento, etc. Los problemas personales se agravan con el tiempo y extienden su influencia a la familia, a los amigos y al entorno social (Del Pozo, 2002; Moisé, 2000). Desde esta perspectiva se demandan acciones eficaces para reforzar la estabilidad emocional como medidas para afrontar el desempleo: "Estas intervenciones tendrán que dirigirse a romper la espiral de decadencia personal y a superar déficits previos facilitando un aumento de la probabilidad de inserción" (Pérez, 1996, 33).

A la luz de lo expuesto, los servicios públicos de empleo precisan innovar para conseguir un aumento en la eficacia de sus acciones. En este artículo se presenta una propuesta de intervención que parte del conocimiento y desarrollo de las competencias emocionales de los desempleados. Se trata de la aplicación del QDE-R (Cuestionario de Desarrollo 
Emocional para adultos, en su versión reducida) que utilizado en el marco de los procesos de orientación profesional y formación permite determinar el nivel de competencia emocional de las personas que buscan empleo y ayuda a orientar las intervenciones necesarias para el desarrollo de las mismas. La experiencia se inició en 2007 en el Centro IGNOVA Ocupació de l'Ajuntament d'Igualada y continua actualmente en colaboración con el (GROP) Grup de Recerca en Orientació Psicopedagògica de la Universidad de Barcelona.

Siguiendo el marco teórico de referencia (Bisquerra y Pérez, 2007) se entiende por educación emocional aquella acción educativa que tiene como objetivo desarrollar las competencias emocionales, que contribuirán a un mayor bienestar personal y social. Las competencias emocionales incluyen el conjunto de conocimientos, capacidades, habilidades y actitudes necesarias para comprender, expresar y regular de forma apropiada los fenómenos emocionales y se estructura en cinco dimensiones: conciencia emocional, regulación emocional, autonomía emocional (autogestión), competencia social y competencias para la vida y el bienestar, que a la vez se subdividen en componentes más concretas. En el concepto de competencia se integra el saber, saber hacer y saber ser.

\section{Método}

\section{Instrumento}

A partir del modelo teórico anterior y de investigaciones previas desarrolladas por el GROP se optó por aplicar el (QDE-R) cuestionario de desarrollo emocional para adultos en la versión reducida.

El QDE-R consta de 27 ítems con formato de respuesta tipo Likert, con once opciones de respuesta de cero a diez. El cuestionario aporta información sobre las cinco dimensiones de la competencia emocional además de una puntuación global. El QDE-R se elaboró seleccionando los ítems con mayor poder de discriminación del cuestionario de desarrollo emocional para adultos en la versión extensa. Su índice de consistencia interna (alfa de Cronba$\mathrm{ch}=0,85)$ puede considerarse óptimo. Se puede consultar el QDE-R en http://stel.ub.edu/ grop/webfm_send/27/QDE-R.

\section{Muestra}

La muestra estaba formada por un grupo de 46 personas usuarias del Servicio Local de Ocupación del Ayuntamiento de Igualada. Se trata de un grupo heterogéneo. La mayoría de las personas eran mujeres (42), en edades comprendidas entre los 19 y 64 años con una edad promedio de 44 años. Todas ellas desempleadas y en proceso de búsqueda de empleo. El 28,4 \% con una antigüedad mayor a un año por lo que pueden considerarse desempleados de larga duración. La mayoría dispone de experiencia laboral previa en sectores laborales diversos. Su formación académica es variada: el 58,7\% tienen un nivel de estudios básicos o inferior y un tercio estudios medios. Todas se encontraban realizando cursos de formación ocupacional en nuevas tecnologías o en servicios de atención a las a personas. 


\section{Procedimiento}

Durante los meses de junio y julio del 2007 se pasaron los cuestionarios, de forma individual y totalmente voluntaria. La evaluación diagnóstica junto al análisis de los resultados permitió iniciar el proceso de orientación y la adaptación de las intervenciones a realizar con los usuarios participantes.

\section{Resultados}

En la tabla de resultados que a continuación se presenta, se pueden observar las puntuaciones promedio de las respuestas dadas al cuestionario para cada una de las cinco dimensiones y en el total de la prueba. Las puntuaciones oscilan entre 0 y 10 , interpretándose el 0 como una falta total de competencia en la dimensión y el 10 como un dominio absoluto de la misma. Así pues las puntuaciones más altas son indicadores de mayor dominio y las más bajas indicadores de menor dominio.

El análisis de estos resultados permite afirmar que nos encontramos con un grupo cuya competencia emocional se sitúa en un nivel medio disponiendo de un potencial de mejora o desarrollo global de 3'85 puntos. No obstante, el grupo muestra una indudable heterogeneidad evidenciada por una gran diferencia entre el desarrollo emocional de los usuarios con menor puntuación (3’27) y mayor puntuación (8’31). Estos datos ponen de relieve que será preciso además de adaptar la intervención formativa grupal a la necesidad media del grupo, tomar en consideración las diferencias individuales en las acciones de orientación profesional personalizada.

TABLA 1. Puntuaciones de competencia emocional en el QDE-R.

\begin{tabular}{|l|l|c|c|c|c|c|c|}
\hline \multicolumn{2}{|c|}{} & $\begin{array}{c}\text { Total } \\
\text { QDE-R }\end{array}$ & Conciencia & Regulación & $\begin{array}{c}\text { Competencia } \\
\text { Social }\end{array}$ & Autonomía & $\begin{array}{c}\text { Comp. para } \\
\text { la Vida y el } \\
\text { bienestar }\end{array}$ \\
\hline \multirow{2}{*}{$N$} & Válidos & 46 & 46 & 46 & 46 & 46 & 46 \\
\cline { 2 - 8 } & Perdidos & 0 & 0 & 0 & 0 & 0 & 0 \\
\hline Media & 6,1455 & 6,6051 & 5,4261 & 6,4652 & 5,3870 & 6,7522 \\
\hline Desv. típ. & 1,12733 & 1,86665 & 1,38923 & 1,84514 & 1,29916 & 1,68875 \\
\hline
\end{tabular}

A nivel grupal, se pueden extraer algunas orientaciones. En primer lugar se apunta la necesidad de priorizar el desarrollo de las dimensiones de autonomía y regulación emocional. Partiendo del análisis de cada una de las dimensiones se pueden realizar las siguientes consideraciones:

La dimensión de autonomía emocional es la que presenta un mayor potencial de desarrollo. En este sentido, sería necesario ya sea en los procesos de orientación profesional o de formación, incidir en el aumento de la autoestima, el desarrollo de una actitud positiva delante de la vida, la autorresponsabilización en el proceso de búsqueda de empleo, la práctica del análisis crítico de las normas sociales, y la capacitación para la búsqueda de ayuda y recursos, así como reforzar la autoeficacia personal. 
La segunda dimensión a desarrollar por este grupo de desempleados es la regulación emocional. Actualmente, en los servicios de empleo, observamos con demasiada frecuencia en estas personas emociones como la ira y la agresividad. Conviene diseñar acciones que ayuden a la persona a tomar conciencia de estas reacciones y de sus consecuencias negativas ante el proceso de búsqueda de empleo, ofrecerle formación para el desarrollo de habilidades de afrontamiento para generar emociones positivas.

La competencia social es la tercera dimensión con mayor posibilidad de desarrollo. La mejora de esta dimensión pasaría por trabajar las habilidades para compartir emociones, el comportamiento prosocial, la asertividad, la cooperación, la prevención y resolución de conflictos.

En la dimensión de consciencia emocional el grupo tiene un nivel considerable, este dato es muy satisfactorio, pues es el requisito previo para poder iniciar la dimensión de regulación emocional.

Por último la dimensión de competencias para la vida y el bienestar ha obtenido una puntuación bastante elevada por parte del grupo $(6,7)$. Este dato pone de manifiesto su capacidad para iniciar el proceso de búsqueda de empleo.

Partiendo de los resultados grupales obtenidos en el QDE-R, se pueden diseñar programas de educación emocional a medida, para incorporarlos de forma habitual en la formación ocupacional. Disponemos ya de otras experiencias en este sentido (Ribera y Pérez, 2009). Así tal y como se trabajan actualmente los módulos de inserción laboral y técnicas de búsqueda de empleo, sensibilización medioambiental, e igualdad de oportunidades, veríamos muy oportuno integrar en el módulo de inserción laboral y técnicas de búsqueda de empleo el desarrollo de las competencias emocionales.

\section{Conclusiones}

La introducción del diagnóstico y desarrollo de las competencias emocionales en los procesos de búsqueda y formación para el empleo se manifiestan como un aspecto clave a tener en cuenta para mejorar las capacidades del usuario en el afrontamiento eficaz de las situaciones transitorias de desocupación. Esto conlleva una renovación de las competencias de los técnicos de los servicios públicos de empleo.

Tanto en las tutorías individualizadas como en los procesos de capacitación y búsqueda de empleo, el diagnóstico de las competencias emocionales deviene un punto de partida para ayudar al usuario a desarrollar una actuación más positiva en el acceso y mantenimiento de un puesto de trabajo. La educación emocional ofrece al desempleado una mayor conciencia de sus posibilidades — puntos fuertes - y de sus carencias entendidas como potencialidad de desarrollo. Así la orientación profesional se convierte en un proceso de fortalecimiento personal del usuario - empowerment - en el proceso de inserción laboral.

El intercambio de buenas prácticas como la experiencia presentada en este artículo contribuye a la concienciación y de las administraciones públicas (estatales y autonómicas) y fundamentalmente de los servicios públicos de empleo de la importancia de la incorporación de la gestión de competencias emocionales en las metodologías utilizadas en los procesos de orientación y formación para el empleo. 
No se puede obviar que en los procesos de selección de personal, consultorías y empresas valoran de forma muy destacada las competencias socio-emocionales de los futuros empleados.

\section{Referencias bibliográficas}

Alberici, A. y Serreri P. (2005). Competencias y formación en la edad adulta. Balance de competencias. Barcelona: Laertes.

Bisquerra, R. y Pérez, N. (2007). Las competencias emocionales. Educación XXI. 10. 61-82.

Cherniss, C. (2000). Social and Emotional Competence in the Workplace. En R. Bar-On, y J. D. Parker, (Ed.). The Handbook of Emotional Intelligence. (pp. 433-459). San Francisco: Jossey-Bass.

Del Pozo J. A.; Ruiz, M. A. y San Martín, R. (2002). "Efectos de la duración del desempleo entre los desempleados". Psicothema, 2, (14), 440-443.

Fernández Vázquez, C. (2005). Orientación profesional e inteligencia emocional. Un análisis de competencias emocionales en desempleados recién titulados y urbanos durante el periodo 20032005 en la provincia de Zaragoza. Instituto Aragonés de Empleo. Disponible en: http://portal. aragob.es/pls/portal30/docs/FOLDER/RED_ORIENTACIONAL/DIRECTORIOS/ARTICULOS/ INFORME+MACA.PDF [Consulta: 2007, $\overline{5}$ de septiembre].

Hué, C. (2003). Las emociones como herramienta en la orientación profesional. Universidad de Zaragoza. Disponible en: http://portal.aragon.es/portal/page/portal/RED_ORIENTACIONAL/ CONTENIDOS/MATERIALES_DIDACTICOS/JORNADAS/TALLER+EMOCIONES+Y+ SENTIMIENTOS+EN+LA+ORIENTACI\%C3\%93N.PDF [Consulta: 05 de septiembre de 2007]

Le Boterf, G. (2001). Ingeniería de las competencias. Barcelona: Gestión 2000.

Moisé, C. (2000). Trabajo, desempleo e impacto subjetivo. En I. Cortazzo y C. Moise (Comp). Estado, salud y desocupació, 121-147. Buenos Aires: Paidós.

Pérez Escoda, N. (1996). Programa de formació per a la inserció laboral. Barcelona: CEAC.

Ribera, A. y Pérez, N. (2009). Desenvolupament de competències emocionals per a la inserció laboral de dones altament qualificades en el projecte Atenea. Comunicación presentada en las V Jornadas de Educació Emocional: "La Intel-ligència emocional a les organitzacions". Barcelona, marzo (paper).

Valls, F. (2007). Inteligencia emocional y asesoramiento vocacional y profesional: usos y abusos. Revista electrónica de investigación psicoeducativa, 11, 5 (1), 179-200.

Fecha de recepción: 05-05-2009

Fecha de revisión: 08-06-2009

Fecha de aceptación: 21-10-2009 